\title{
Assaporare la Tradizione: Cibo, Identità e Senso di Appartenenza nella Letteratura Migrante
}

\author{
VERA HORN ${ }^{1}$
}

ABSTRACT: Come sostiene Vito Teti, il cibo per l'immigrato è una forma di difesa di un'identità culturale e riflette il bisogno di riconoscersi e di aggregarsi. Il bisogno di trovare senso e posto in un nuovo luogo vengono segnati da un attaccamento ai cibi perduti, con un senso di sacralità che accompagna il pasto. A partire dalla premessa di Teti, verranno presi in considerazione i romanzi Volevo diventare bianca di Nassera Chohra (1993) e Scontro di civiltà per un ascensore a Piazza Vittorio di Amara Lakhous (2006) e i racconti Curry di pollo di Laila Wadia; Salsicce di Igiaba Scego 
e Il cuoco di Arbëria di Carmine Abate. Tali testi impongono uno sguardo determinante sul cibo come un indice culturale che può rappresentare o costruire l'identità o determinare l'appartenenza ad una certa comunità, così come rifiutare decisamente qualsiasi contaminazione con la cultura della società ospitante o tentativo di assimilazione o, diversamente, offrire un punto di vista fondato sull'ibridismo; inoltre può favorire la formazione di stereotipi. In questo modo vengono definite prospettive diverse di costruzione dell'identità, sintetizzate da Stuart Hall nel binomio traduzione/ tradizione.

PAROLE CHIAVI: migrazione; identità; cibo; appartenenza; ibridismo.

RESUMO: Como afirma Vito Teti, a comida para o imigrado é una forma de defesa de uma identidade cultural e reflete a necessidade de reconhecerse e de agregar-se. A necessidade de encontrar sentido e lugar em um novo espaço são marcados pelo apego às comidas perdidas, com um sentido de sacralidade que acompanha a refeição. A partir da premissa de Teti, se fará a leitura dos romances Volevo diventare bianca, de Nassera Chohra (1993) e Scontro di civiltà per un ascensore a Piazza Vittorio, de Amara Lakhous (2006) e dos contos Curry di pollo de Laila Wadia; Salsicce de Igiaba Scego e Il cuoco di Arbëria de Carmine Abate. Tais textos impõem um olhar determinante sobre a comida como um índice cultural que pode representar a construção da identidade ou determinar o pertencimento a certa comunidade, assim como um modo de recusar decididamente qualquer contaminação da cultura da sociedade que o hospeda ou qualquer tentativa de assimilação ou ainda um modo de oferecer um ponto de vista fundado sobre o hibridismo; além disso, pode favorecer a formação de estereótipos. Desse modo são definidas algumas perspectivas diferentes de construção de identidade, sintetizadas por Stuart Hall no binômio tradução/tradição. PALAVRAS CHAVE: migração; identidade; comida; pertencimento; hibridismo. 
ABSTRACT: According to Vito Teti, food for the immigrant is a way of defending cultural identity and it reflects the need to recognize and unite. The need to find meaning and place in a new land are marked by an attachment to the lost food, with a sense of sacredness that accompanies the meal. From Teti's premise, we will consider the novels Volevo diventare bianca byNassera Chohra (1993) and Scontro di civiltà per un ascensore a Piazza Vittorio by Amara Lakhous (2006) and the tales Curry di pollo by Laila Wadia; Salsicce by Igiaba Scego and Il cuoco di Arbëria by Carmine Abate. These books require a crucial look at food as a cultural indication that can represent or built or the identity or determine membership to a particular community, as well as to refuse any contamination with the culture of the host society, or attempt to assimilate, or otherwise provide a hibrid point of view; besides encouraging the formation of stereotypes. So, different perspectives are defined sense of identity, summarized by Stuart Hall combining translation / tradition.

KEYWORDS: migration; identity; food; appearance; hydridism. 
When Irie stepped over the threshold of the Chalfen house, she felt an illicit thrill, like a Jew munching a sausage or a Hindu grabbing a Big Mac. She was crossing borders, sneaking into England.

(Zadie Smith, White teeth)

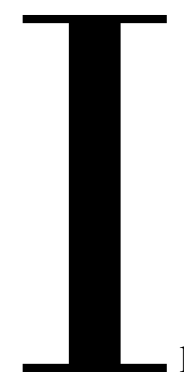

1 cibo, come straordinario indice culturale, costituisce un potente strumento dell'identità e, come afferma Massimo Montanari, è "il primo modo per entrare in contatto con culture diverse": mangiare il cibo altrui è più facile, almeno in apparenza, che decodificarne la lingua; inoltre, più della parola, il cibo si presta a mediare fra culture diverse, aprendo i sistemi culturali (le "cucine") agli incroci e alle contaminazioni.

Quando si tratta delle culture alimentari, spesso vengono chiamate in causa le nozioni di identità e di scambio, talvolta contrapposte, come se lo scambio - "confronto fra identità diverse" - fosse un ostacolo all'identità, e cioè al mantenimento del patrimonio culturale di ciascuna società. L'assunzione di questa prerogativa, che spesso si sposa alla diffidenza verso il diverso, alla paura della contaminazione e, quindi, a forme più o meno esasperate di chiusura e di intolleranza, vede spesso chiamata in causa la storia come luogo restauratore delle "radici", delle "origini" più o meno mitiche interessate alla "conservazione della propria identità". Invece la storia, avverte Montanari, ci mostra esattamente il contrario: 
che le identità non sono realtà metafisiche (lo "spirito dei popoli") e neppure sono inscritte nel patrimonio genetico di una società, ma si modificano e si ridefiniscono incessantemente, adattandosi a situazioni sempre nuove determinate dal contatto con culture diverse (MONTANARI, 2004, p. 154).

Le nozioni di identità e scambio postulate da Montanari introducono comportamenti alimentari che in un contesto immigratorio sono fortemente collegati al senso di identità, di luogo e di appartenenza. Il migrante, espatriato dal proprio suolo, spesso raggiungibile soltanto tramite la memoria, sperimenta un senso di appartenenza precario: egli non appartiene più alla terra di origine, ma neanche a quella di arrivo. Questo vivere tra due luoghi e allo stesso tempo dentro e fuori di una situazione

significa vivere all'intersezione tra storie e memorie, sperimentando sia la loro dispersionepreliminare sia la successiva traduzione in nuovi e più ampi assetti lungo percorsi emergenti. (CHAMBERS, 1996, p. 10)

La questione è complessa e comprende diversi punti di riferimento (lingua, religione, musica, arte, abbigliamento), tra cui quello del cibo, associato, in questo caso, al senso dell'identità e al bisogno di riconoscersi e di identificarsi. Il migrante, immerso in un contesto alieno, dalle connotazioni culturali, spaziali, umane talvolta radicalmente diverse dalle sue, è portato ad un continuo riconoscimento di elementi di somiglianza e di differenza rispetto al luogo di origine, che, se tradotto in comportamenti alimentari, significa preservare o cambiare, situazioni che Vito Teti individua nei comportamenti alimentari dei migranti come di continuità e mutamento:

L'emigrazione è stata considerata, a seconda dei diversi punti di vista, ora come elemento di "conservazione" ora come fenomeno di "rottura" dell'ordine tradizionale e gli emigrati sono stati descritti ora come inguaribili nostalgici ora come "ribelli" sempre irrequieti e insoddisfatti. In realtà nel movimento emigratorio continuità e mutamento, tradizione e innovazione, conservazione e trasformazione convivono, coesistono, s'incontrano e concorrono alla costruzione di un "ordine nuovo" rispetto 
a quello di origine, che comunque, reale o immaginario, vero o inventato, resta un ineludibile punto di riferimento e di "ritorno". (TETI, 2001, pp. 576-577)

L'emigrazione rappresenta un elemento di "trasformazione dei consumi e delle abitudini alimentari", da una parte, e del "conservazionismo culinario" e della "nostalgia alimentare" dall'altra (TETI, 2001, pp. 577-586). Il contatto con nuove abitudini e nuove realtà porta ad un cambiamento dei comportamenti alimentari; il risultato degli incontri, degli scambi e delle reciproche influenze, in questo caso, può essere una cucina dai tratti ibridi, emblema di una società in trasformazione. Tuttavia, ciò non significa che il semplice acquisto saltuario e casuale di prodotti cosiddetti esotici o etnici nei supermercati più forniti rientri in questo paradigma: si tratta, in questo caso, di una forma superficiale di pluralismo. La cucina della tradizione rimanda invece alla necessità di ricreare il proprio luogo.

La ricostruzione delle abitudini alimentari nel luogo d'arrivo è, inoltre, da associare alla nostalgia per i cibi perduti, corollario naturale della perdita del luogo (casa) e dei legami sociali/affettivi.

Nella letteratura della migrazione, il cibo viene spesso trattato come indice di identità e di cultura, sotto gli aspetti della continuità/differenza, o della ibridazione o assimilazione. Nei paragrafi seguenti, cercheremo di analizzare in che modo ciò avviene, mediante l'esame di testi in cui emerge uno stretto nesso tra il migrante e il cibo e le questioni identitarie che ne derivano.

Il racconto Salsicce di Igiaba Scego, scrittrice di origini somale, presenta un dilemma identitario trasposto in una questione alimentare. Nella trama, la protagonista, una musulmana sunnita di origini somale e nata in Italia, decide di comprare salsicce in piena vigilia di Ferragosto: cucinarle e poi mangiarle sarebbe un modo di dimostrare la sua identità italiana e di risolvere il suo conflitto identitario. Tuttavia, sin dall'inizio, lei non è convinta della bravata, come la chiama, e si riferisce alle salsicce come "pacco pieno di salsicce impure" e "l'impudico pacco" (SCEGO, 2006, pp. 25-26) in un preciso riferimento alle salsicce come cibo haram (impuro) nella concezione islamica.

La protagonista in realtà si sente divisa tra un'identità italiana e una somala, a cui 
non sa come rapportarsi. Le salsicce sarebbero la soluzione eventuale del problema:

Se mi ingoio queste salsicce una per una, la gente lo capirà che sono italiana come loro? Identica a loro? (SCEGO, 2006, p. 25)

La questione delle identità plurali che abitano spazi interstiziali viene descritta nel testo di Scego come risposta possibile, ma non conclusiva, alle insicurezze identitarie della protagonista. In realtà, le è chiara la sua doppia appartenenza culturale. Entrambe le dimensioni, italiana e somala, fanno parte della sua identità, senza che l'una possa prevalere sull'altra o assimilarsi all'altra, negandola, come è chiaro dalla conclusione con l'esperienza delle salsicce. Il rifiuto di ingurgitare le salsicce è un atto simbolico; la protagonista non riconosce un'appartenenza unica, imposta da schemi sociali o legislativi:

Infilzo con la forchetta la salsiccia più piccola, l'avvicino al naso. AGHHHH, puzza! Chiudo gli occhi e avvicino l'immondità alla bocca. Comincio a sentire un sapore acido come vomito. Allora è questo il gusto della salsiccia, vomito? Poi qualcosa mi bagna il petto ed è allora che apro gli occhi. Con stupore noto di aver vomitato la colazione della mattina, una tazza di creali con latte freddo e una mela. E la salsiccia? Dov'è la salsiccia? È ancora infilzata tutta intera sulla forchetta. Non ho fatto in tempo a metterla in bocca, il vomito l'ha preceduta. (SCEGO, 2006, pp. 31-32)

L'io narrante ritrova, in una definizione ibrida e plurale della propria identità, una risposta agli schemi mentali dell'altro che la volevano costringere ad una scelta tra i due mondi a cui appartiene. Ciò non significa una semplificazione del problema, bensì il doversi confrontare con una continua negoziazione tra le culture, senza obbedire a standard identitari rigidi condizionati da un luogo o da paradigmi di classe, genere, sesso, etnia, razza, rappresentati idealmente dalle salsicce:

Guardo le salsicce e le getto nell'immondezzaio. Ma come ho potuto solo pensare di mangiarle? Perché voglio negare me stessa, solo per far contenta una signora butterata con la voce da travestito? O far contenti 
i sadici che hanno introdotto l'umiliazione delle impronte? Sarei più italiana con una salsiccia nello stomaco? E sarei meno somala? O tutto il contrario?

No, sarei la stessa, lo stesso mix.(SCEGO, 2006, p. 35)

Il racconto Curry di pollo della scrittrice indiana Laila Wadia, vincitore della edizione 2004 del concorso Eks\&Tra, raffigura il dramma opposto di Salsicce. La protagonista Anandita è una ragazzina di sedici anni nata in Italia da genitori indiani profondamente radicati nella loro cultura. Lei, al contrario, desidera a tutti costi sembrare italiana, essere italiana, assimilandosi alla cultura dominante:

Ho sedici anni e vivo a Milano. Non posso non andare in discoteca, non posso non farmi il piercing, non posso non avere un ragazzo - lo fanno e ce l'hanno tutte le mie amiche. (WADIA, 206, p. 39)

I contrasti tra la cultura alla quale desidera di appartenere e quella di origine dei genitori sono evidenti:

I miei sono dei Flintstones indiani che pensano di vivere ancora in una capanna di fango nell'oscuro villaggio di Mirapur, nell'India centrale, con le loro due mucche e le tre capre. Invece, da più di vent'anni abitano qui nel centro di Milano. (WADIA, 2006, p. 39)

Il simbolo di questo contrasto tra culture è proprio il curry di pollo, il piatto indiano di cui va fiera la madre di Anandita, la cui ricetta ha ereditato a sua volta dalla suocera, che "sapeva fare il miglior curry di pollo di tutto il distretto di Mirapur". Nel racconto, il curry di pollo diventa il simbolo della rappresentazione di un'identità che la protagonista vuole negare: è il tratto della differenza e può essere assunto a scopi esclusionisti.

La situazione diventa più marcatamente contrastante quando si presenta un'occasione conviviale: il ragazzo italiano di Anandita è invitato a cena. Lei insiste con la madre che per l'occasione non prepari il curry di pollo, bensì un piatto italiano come 
le penne al pomodoro. Durante la cena, che avviene in un clima che rasenta il farsesco, il padre di Anandita, dopo aver assaggiato la pasta "come un pellicano farebbe con una rana saltellante", definendola "tubi di gomma", chiama in causa il curry di pollo. L'ironia del racconto si fa più viva quando il ragazzo, incalzato dalle domande del genitore, ammette di apprezzare il curry e di averlo mangiato in una pizza "funghi, panna e curry" e in un risotto in busta "ai gamberetti e curry" a cui va aggiunto "un cucchiaio di parmigiano e una noce di burro". L'innovazione moderna, per il genitore indiano, è messa in contrasto con la tradizione, a discapito della prima:

\footnotetext{
"Devo far provare a questo giovanotto la sublimazione dei sensi. Devo fargli dimenticare gli orrori della pizza al curry o del risotto al curry in busta. Sì, mia moglie segue la stessa ricetta che usava mia madre, pace all'anima sua. Faceva il miglior curry di pollo dell'intero distretto di Mirapur. Prima macinava tre tipi di peperoncini con le altre spezie - senape, coriandolo, cardamomo, papavero, cannella, chiodi di garofano -, poi li friggeva con la cipolla e l'aglio e infine ci aggiungeva un pomodoro, il latte di cocco e il pollo (...)" (WADIA, 2006, p. 51)
}

La ricchezza di dettagli del piatto, che ha una storia e una cultura di supporto, nonché la descrizione vivida della sua preparazione contrastano visibilmente con l'inadeguatezza dei risotti in busta a cui vanno aggiunti pochi ingredienti, non supportati da alcuna tradizione o pregio che non sia quello della rapida preparazione. Sono due culture in realtà che si confrontano per l'immigrato indiano che cerca di restaurare nel presente dislocato e deterritorializzato la purezza di un passato al quale non può più fare ritorno. Il curry di pollo funziona come una specie di comunità immaginata, un anello di congiunzione che collega i membri della famiglia/comunità. Nel comportamento del genitore indiano è evidente il principio dell'identità, definito da Massimo Montanari come la salvaguardia del patrimonio culturale che ogni società riconosce nel proprio passato, contro la possibilità dello scambio, inteso come confronto tra identità diverse. Da qui la chiusura della famiglia nelle proprie tradizioni:

Nonostante la lunga permanenza in Italia, mamma si veste sempre all'indiana, sfoggiando un sari sgargiante dopo l'altro, si pettina sempre 
all'indiana, cucina sempre all'indiana, parla sempre indiano. (WADIA, 2006, p. 40)

Il curry di pollo diventa, nel racconto, il tratto identificante principale di un'identità alla quale la protagonista non desidera appartenere, a cui si aggiungono altri tratti identitari che lei cerca di cancellare (è emblematico il gesto di gettare il pane indiano nella spazzatura), adottando invece quelli della cultura dell'ambiente in cui si trova:

Non sanno neanche che ho un piercing all'ombelico, che quando dico che vado a studiare da Samantha la domenica pomeriggio in verità andiamo in discoteca, che butto via il sacchetto con il pane indiano farcito di verdure strangolate nell'olio e nelle spezie che la mamma mi fa portare a scuola per merenda. (WADIA, 2006, p. 45)

Nel racconto si confrontano due prospettive diverse di costruzione dell'identità. La prima è rappresentata dai genitori di Anandita che, pur inseriti in un'altra società da lungo tempo, resistono a tutte le pressioni della contaminazione culturale e dell'influenza della cultura dominante sulla loro. La seconda è quella di Anandita stessa, che, pur vivendo in un ambiente familiare che preserva la cultura d'origine e conserva i valori originari, tende a identificarsi con la cultura egemone, a tutti gli effetti il suo unico punto di riferimento:

Non ribadirò il fatto che sono nata e cresciuta in Italia, che in Italia nessuno si sogna di far sposare una figlia di sedici anni, e che non voglio sposarmi con un mungitore di vacca o con il campione degli arrampicatori di cocco di Mirapur. Mi sposerò solo con Marco, il mio bel ragazzo dagli occhi di zaffiro e i capelli di Brad Pitt. Non miagolerò che non voglio mettermi il vestito indiano come fa la mamma. (A Marco piace la minigonna). Che non voglio mettermi il puntino sulla fronte come fa la mamma. (Marco dice che ho una pelle vellutata come un camoscio.) Che non voglio portare i sandali infradito. (Marco adora i tacchi alti.) (WADIA, 2006, p. 44)

Volevo diventare bianca, il romanzo autobiografico della scrittrice marsigliese di 
origini saharawi Nassera Chohra, pubblicato nei primi anni della letteratura migrante (1993), racconta le forme del pluralismo culturale da una prospettiva diversa rispetto a Curry di pollo, in cui la protagonista, malgrado viva di fatto in una situazione plurale, preferisce negare la sua identità indiana in favore di quella italiana, della quale assume i connotati. L'opera di Chohra narra la formazione della protagonista Naci, nata e cresciuta a Marsiglia da genitori algerini provenienti dalle tribù saharawi. Naci trascorre l'infanzia nelle banlieue marsigliesi, "vera e propria torre di Babele, un miscuglio di razze, culture e religioni" (CHOHRA, 1993, p. 9), dove convive con ragazze francesi e figli di immigrati. In questo romanzo è evidente l'importanza del cibo come indice culturale, come avverte Gian Paolo Biasin nell'articolo Gelato e peperoncino (1995):

il cibo è certamente uno dei più piacevoli e dei più ricchi di significati e di potenzialità, perché il cibo è un connotatore sociale, psicologico e morale di grande immediatezza e vivezza, dato che il linguaggio stesso abbonda di usi metaforici che partendo dal riferimento alimentare lo espandono ad altre aree, come l'evangelico "avere fame e sete di giustizia" o il comunissimo "divorare un romanzo dalla prima all'ultima pagina". (BIASIN, 1995, p. 103)

Nel romanzo, il cibo si collega alle contraddizioni identitarie della formazione di Naci, nera, nata in Francia da genitori immigrati, musulmana, che aspira a diventare bianca (come riportato nel titolo, motivo di un divertente quanto drammatico episodio con la candeggina), francese, cattolica. Durante l'infanzia della protagonista, l'associazione tra il cibo e le questioni religiose offre spunto per una riflessione sull'identità e l'appartenenza. Quando le compagne di scuola si preparano alla prima comunione, nasce nella protagonista il desiderio di poter vivere questa esperienza, ma la sua religione musulmana lo vieta. Durante il ricevimento per la prima comunione di una delle compagne, Naci decide quindi di convertirsi alla religione cattolica:

Oltre ai dolci, sul buffet c'erano anche salame, salsicce, prosciutto e vino rosso: un concentrato di peccati gravissimi, secondo il Corano. Li osservavo a distanza cercando di non attirare l'attenzione. Loro non sapevano che quel giorno la mia vita sarebbe cambiata. Quando mi fossi completamente confusa tra la folla e nessuno avesse più badato a me, 
allora avrei potuto dare inizio alla mia conversione. Come? Mangiando il salame e bevendo il vino, naturalmente. A sentir tutti, era solo quella la differenza tra noi e i cattolici! (CHOHRA, 1993, pp. 66-67)

Lei si comporta in questo episodio come l'io narrante di Salsicce, credendo così di poter acquisire un'altra identità per via del cibo. Tuttavia, a differenza del primo racconto, la protagonista ingoia veramente i cibi vietati ai musulmani: mangiando quello che mangia l'altro, sono l'altro:

Il salame aveva un sapore salato e il vino aveva un po' il gusto dell'aceto rosso al quale era stato aggiunto dello zucchero. Li avevo ingurgitati in un istante, senza quasi masticare. Ripetei quel gesto più volte, come fossi una ladra. Ogni boccone era sempre più grande e ogni sorso di vino abbondante, finché ritenni che potesse bastare. A quel punto dovevo per forza essere diventata cattolica. (CHOHRA, 1993, p. 67)

Nel gesto di ingerire i cibi vietati Naci cerca di appropriarsi della cultura, in questo caso religiosa, dell'altro, e rientrare nei canoni delle compagne di scuola, allontanandosi così dalla cultura dei genitori, come spiega alla maestra, perplessa di fronte alla comunicazione di Naci sulla sua prossima prima comunione:

"Sa, signora maestra, io sono diversa dai miei genitori, io mangio il salame e bevo il vino, perciò sono cattolica." (CHOHRA, 1993, p. 69)

1 cibo è spesso associato all'osservanza di principi religiosi; si pensi ad esempio ad alcuni precetti basilari come il rituale della comunione o l'astinenza della carne per i cattolici nel venerdì santo, le restrizioni alimentari dei musulmani o degli ebrei, il divieto della carne di mucca per gli induisti. Il collegamento tra cibo e religione è uno dei risvolti della questione nel romanzo, che si sviluppa in altre dinamiche. Nell'articolo sopracitato Gelato e peperoncino (1995), Gian Paolo Biasin si occupa del romanzo di Chohra, definendolo "un'autobiografia e una testimonianza, ma che si può leggere come vero e proprio 'romanzo di formazione' contemporaneo", in cui 
la protagonista viene "esposta a tutte le contraddizioni e conflitti insiti nella sua situazione - dall'incontro con il razzismo alla presa di coscienza della sua condizione femminile, dall'Islam al Cristianesimo, dal 'terzo mondo' all'occidente". Il critico sottolinea la golosità della protagonista come "elemento fondamentale e preminente delle esperienze narrate" nel romanzo (BIASIN, 1995, p. 105).

Nell'infanzia di Naci il gelato resta un simbolo della separazione tra la classe immigrata e povera a cui appartiene, la sua pelle nera, e quella delle sue compagne di gioco, bianche e francesi, come Corinne, la vicina di casa nella cui famiglia ognuno si può permettere un gelato (nella numerosa famiglia di Naci un gelato deve bastare per sette), e Michelle, l'altra vicina di casa, a cui Naci ruba un gelato.

Il castigo inflitto a Naci dalla madre per l'episodio del furto introduce un altro elemento culinario: il peperoncino, utilizzato in questo caso non per condire le pietanze di origine algerina o saharawi, ma come una punizione terribile destinata a provocare bruciore nelle parti intime:

Tutta nuda e con le gambe aperte sembravo un pollo da condire prima di metterlo al forno. Il peperoncino, strofinato ripetutamente sul mio sesso, bruciava da morire. Era come se prendessi fuoco dal di dentro. (CHOHRA, 1993, p. 23)

Oltre all'aspetto sociale rappresentato dal gelato, che riguarda i conflitti di classe nella cintura urbana di una Marsiglia multietnica, sia il gelato che il peperoncino hanno nel testo un carattere culturale nell'affermare le espressioni del piacere e della punizione legate ai comportamenti alimentari delle culture rappresentate, quella occidentale francese e quella algerina saharawi.

In sintonia con la coppia indiana del racconto Curry di pollo, il collegamento tra identità e cibo proposto nel romanzo Scontro di civiltà per un ascensore a Piazza Vittorio (2006), dello scrittore algerino Amara Lakhous, tramite il personaggio Parviz recupera nella nostalgia la dimensione identitaria perduta. Parviz, iraniano di Shiraz, è un esempio chiaro della non integrazione. Il personaggio vive a Roma, dove svolge lavori saltuari come aiuto cuoco o lavapiatti, che finisce per abbandonare presto, non 
solo per il suo vizio di bere sul lavoro, ma soprattutto per la sua antipatia verso la cucina italiana, che lo porta a ignorare il nome delle diverse erbe e degli ingredienti necessari alla preparazione dei piatti, rendendo in questo modo la sua presenza inutile nella cucina di un ristorante. Come cuoco, rimane profondamente legato alla sua cultura gastronomica, che è poi il filo che lo lega alla sua terra, quello che rimane della sua memoria:

Mi metto subito a preparare i vari piatti iraniani, come il gormeh sabzi e il kabab kubideh, i kashk badinjan e i kateh. Gli odori che riempiono la cucina mi fanno dimenticare la realtà e mi sembra di essere tornato nella mia cucina a Shiraz. Dopo un po' il profumo delle spezie si trasforma in incenso, ed è questo che mi fa ballare e cantare come un derviscio, ahi ahi ahi... Così in pochi minuti la cucina si trasforma in una trance sufi. (LAKHOUS, 2006, p. 35)

Come sostiene Vito Teti, il cibo per l'emigrato è una forma di difesa di un'identità culturale e riflette il bisogno di riconoscersi e di aggregarsi. Il bisogno di trovare senso e posto in un nuovo luogo vengono segnati da un attaccamento ai cibi perduti, con un senso di sacralità che accompagna il pasto (TETI, 2001, pp. 579; 590).

Ahmed-Amedeo, il protagonista del romanzo, sostiene, nel suo primo "ululato", che Parviz si rifiuta di imparare la cucina italiana perché ha paura di dimenticare quella iraniana - e cioè, di perdere la sua identità. Parviz è anche l'autore di una divertente condanna ai "pizzadipendenti”. Coerentemente, Parviz è contrario all'omologazione gastronomica e quindi ai "pizzadipendenti": tra i quali si annovera tuttavia anche l'amico Amedeo, cioè l'immigrato Ahmed, con un inconsapevole e rovesciato atteggiamento razzista:

Il mio odio per la pizza non ha paragoni, ma questo non significa che io odi tutte le persone che la mangiano. (...)

Amo molto Amedeo, nonostante sia un pizzadipendente. (...)

La mia preoccupazione è di evitare a tutti i costi le conseguenze dovute alla mia avversione alla pizza. Per esempio, qualche settimana fa mi hanno licenziato dal posto di lavapiatti in un ristorante vicino a piazza Navona quando hanno scoperto che odiavo la pizza. Figli di puttana.

\footnotetext{
2. Gli "ululati" sono le riflessioni del protagonista, che si alternano nel romanzo alle "verità" degli altri personaggi; in realtà sono una sorta di deposizione proprio sul personaggio Ahmed/Amedeo, accusato ingiustamente di un omicidio. Il doppio nome del personaggio è dovuto ad una errata comprensione del nome di Ahmed (il nome reale) dalla parte del barista romano Sandro Dandini: questi lo pronuncia Amed, che diventa presto Amedé e quindi Amedeo per gli altri. Non si tratta però di un semplice cambio di nomi: si sviluppano in questo modo implicazioni identitarie collegate alla questione immigratoria.
} 
Dopo questo scandalo c'è ancora chi sostiene che la libertà di gusto, di espressione, di fede e la democrazia sono garantite in questo paese! Vorrei sapere: la legge punisce chi odia la pizza? Se la risposta è sì, siamo di fronte a un vero scandalo, se è no, allora io ho tutti i diritti di essere risarcito. (LAKHOUS, 2006, pp. 12-13)

Le forme di resistenza alla cultura dell'altro possono essere diverse; per Parviz essa significa un attaccamento esageratamente nostalgico alla propria cultura, con il rifiuto categorico della cultura dell'altro, diventata oggetto di sentimenti di odio (simboleggiato nell'odio alla pizza). Nel rifiutare categoricamente la cucina italiana, rifacendo sempre con uguale passione i piatti della cucina iraniana, Parviz recupera la sua cultura di origine, la sua terra (Shiraz), la sua famiglia, le sue abitudini e tradizioni, e se ne riappropria.

In questo brano, la nostalgia di Parviz, descritta dal protagonista Ahmed/Amedeo, esprime un legame tra il mondo di appartenenza e quello di arrivo. Tuttavia, Parviz non rappresenta semplicemente un banale quanto inopportuno sentimento di nostalgia, assunta a simbolo di un conservatorismo culturale e culinario radicale: nel suo comportamento si riscontrano gli echi della necessità di appaesamento del migrante. Come sottolinea Vito Teti, esso evoca il luogo antropologico (secondo Marc Augé, il luogo antropologico è allo stesso tempo identitario, relazionale e storico ${ }^{3}$ ):

Mangiare come nel luogo d'origine ha in qualche modo contribuito a placare la nostalgia, come se insieme al cibo e alle abitudini alimentari si fossero portati con sé nel nuovo mondo anche la casa, l'orto, i familiari, gli amici. Il cibo evoca e in qualche modo presentifica un luogo antropologico, fatto di parole, memorie, ricordi, storie, persone, relazioni. (TETI, 1999, p. 90)

Le problematiche finora emerse dai testi presi in esame confluiscono nel racconto Il cuoco d'Arbëria di Carmine Abate ${ }^{4}$. Nell'opera di questo scrittore il cibo occupa un ruolo di rilievo; non è occasionale la presenza del cibo nella valigia del migrante che dalla Calabria è costretto a emigrare in Francia (La festa del ritorno, 2004) o in Germania (Il ballo tondo, 1991; Il muro dei muri, 1993; La moto di Scanderberg, 1999;

3. Vedi Augé, M. Nonluogh: introduzione a una antropologia della surmodernità. Trad. D. Rolland. Milano: Eléuthera, 2000.

4. Carmine Abate non è propriamente uno scrittore migrante se intendiamo come facenti parte di questa "categoria" gli scrittori nati altrove che scelgono di scrivere nella lingua italiana, la lingua del paese d'immigrazione; tuttavia, per le tematiche emigratorie delle sue opere, nonché per il suo vissuto (nato in un paese arbëreshe, Carfizzi, nel crotonese, figlio di immigranti, diventa lui stesso un migrante dopo essere partito per la Germania come il genitore, per poi tornare in Italia, dove risiede tuttora), è spesso associato al fenomeno della letteratura migrante. 
Tra due mari, 2002). Nei ritorni temporanei al paese, solitamente estivi (ma anche pasquali o natalizi), il migrante ritrova nella tavola della tradizione un anello di congiungimento con la propria terra.

Le figure femminili delle opere di Abate, le madri, le moglie e le nonne si comportano in questo senso come forze motrici nel tenere insieme tradizioni e famiglia tramite la preparazione e la conservazione del cibo e l'apparecchiamento della tavola. La dimensione del pasto consumato insieme acquisisce un valore sacrale; mangiare insieme rende possibile riconoscersi e riconoscere l'altro. Mangiare alla stessa tavola rafforza il senso d'identità di gruppo e pertanto di appartenenza; si assolve in questo modo un bisogno primario dell'uomo spaesato. Con il ritorno provvisorio, il migrante non solo si riconcilia con la terra d'origine tramite la "riconquistata familiarità con i cibi perduti" di cui parla Teti, ma nei bagagli porta il cibo del paese, solitamente in conserva, nelle terre dell'espatrio, quasi fosse il luogo stesso.

Nel racconto Il cuoco d'Arbëria, gli invitati di un banchetto nuziale assaggiano le numerose pietanze preparate dal cuoco d'Arbëria (il nome indica le comunità originarie delle popolazioni albanesi immigrate in Italia a partire dal XV secolo) secondo la tradizione arbëreshe; esse vengono chiamate con il loro nome originale, a cui segue la traduzione in italiano:

Ma, intanto, dopo gli applausi e gli evviva, tutti si erano buttati a capofitto sugli antipasti e in un batter d'occhio erano spariti gli affettati di capicollo prosciutto salsiccia soppressata, serviti con këpurdha te vajt, funghi sott'olio, ullini të zeza te kripa, olive nere in salamoia, ullinj të shtipur, olive schiacciate con pezzettini di aglio e peperoncino, mallanxhane te uthulla e te vajt, melanzane sott'aceto e sott'olio; ed erano sparite le fette di pane fatto in casa ricoperti con fiq palleti te tigani, bucce di fichi d'India in padella, cotti con melanzane, pomodori e bucce di melone.

Per nostra fortuna, il cuoco d'Arbëria batté le mani e fece entrare cinque donne che portavano in grandi piatti fumanti shtridhëlat, la pasta filata con farina di grano tenero e grano duro, e condita con fagioli bianchi, olio, aglio e peperoncino. (ABATE, 2000, p. 329)

Sono pietanze della tradizione arbëreshe autentica secondo il cuoco d'Arbëria: "la sua cucina era arbëreshe perché lui era arbëreshe": la tradizione culinaria viene utili- 
zzata per definire l'identità. Tuttavia, come si affretta a spiegare l'io narrante, i piatti preparati dal cuoco erano di fatto di tradizione arbëreshe, ma avevano subito delle contaminazioni dalla parte delle cucine meridionali e potevano essere trovati con lievi differenze anche nei paesi litirë (forestieri) del circondario.

Durante il pranzo, il cuoco ricorda gli antenati albanesi del popolo arbëreshe della Calabria, scappati cinque secoli prima alla dominazione dei turchi, che avevano dato luogo alla loro civiltà e alla loro cucina:

Partirono un giorno bello sereno come oggi ma lontano cinque secoli fa, disse l'uomo prima che si cominciasse a banchettare. Partirono per non sottostare ai turchi, con tre galee stracariche, gli antenati nostri d'Albania: una di giovani, l'altra di ragazze, la terza, infine, di pane e vino, cantavano gli antichi. (...) secondo perché non portarono solo pane e vino - e questo io lo so, ne ho le prove qui dentro nello stomaco. Portarono anche olio e olive, farina e miele, uova ricotta e formaggio, mandorle e fichi secchi, fave e ceci, vitellini agnelli e capretti, carciofini finocchio selvatico origano prezzemolo cardi mangerecci basilico alloro ruta aglio e cipolla, prosciutti e salsicce. E tutti questi cibi e altri ancora, li troverete nelle pietanze che le brave zonje di Carfizzi col mio aiuto hanno preparato e che voi gusterete col vino forte di Cirò. (ABATE, 2000, p. 328)

La memoria degli antenati arbëreshe è strettamente associata al cibo, sia perché è un'espressione della loro identità culturale e un modo di preservare il passato, sia perché il fatto di portare i cibi del luogo d'origine in terra straniera rivela i percorsi dell'appartenenza:

La provenienza, l'appartenenza, l'identità delle persone in viaggio sono riconoscibili da quello che mangiano, da come trattano i cibo, dal modo di tenere o tagliare col coltellino il pane o la frutta, dal modo di masticare il cibo, seduti sul proprio sacco, nella sala dove aspettano la nave o il treno, dal bisogno di portarsi appresso riserve o di farsele inviare tramite corriere, come avviene ancora oggi. (TETI, 2001, p. 589)

Il racconto del cuoco d'Arbëria parte dall'emigrazione degli albanesi diretti in Ita- 
lia, ma nella trama si sviluppa un'altra emigrazione, raccontata frequentemente nelle opere di Abate, quella dei calabresi, espulsi dalla loro terra verso i paesi del Nord, nella fattispecie la Germania.

La costrizione ad emigrare porterà in Germania l'io narrante, il genitore ed il cuoco stesso, "partito nei primi anni Sessanta con un contratto di lavoro in tasca". L'io narrante lo ritrova poi casualmente a Hainburg, molti anni dopo, al centro Italiano, "luogo di ritrovo degli italiani della zona", dove il cuoco prepara i piatti della tradizione arbëreshe, tra cui i famosi shtridhëlat del banchetto nuziale, tramite il quale il narratore riconosce il cuoco d'Arbëria della sua infanzia: in Germania aveva lavorato nei cantieri edili e navali, fonderie, ferrovie e perfino in un ristorante, dove si era stancato di "riscaldare spaghetti scotti alla bolognese e pizze margherite". Mentre prepara saucicë me vrokul rrapje (salsiccia tedesca con broccoli di rape conditi con aglio, olio e peperoncino) al Centro Italiano, il cuoco d'Arbëria ammette di avere adeguato il piatto al palato locale. L'utilizzo della salsiccia tedesca nella preparazione di un piatto arbëreshe, condito tuttavia alla maniera tradizionale (aglio, olio, peperoncino) risulta l'emblema di una contaminazione tra culture alimentari che egli stesso ammette:

Comunque io, per me, mi sono adeguato. Tanto per dire: mangio anche Wurstel con peperoncino, sono diventato patataro più dei tedeschi. Kartoffelfresser, come dicono loro, patate cotte, ma condite a modo nostro, con aceto, olio, peperoncino tritato, aglio e bevo birra a volontà. Ma questo non significa intedescarsi, significa essere un uomo con un palato diviso, o forse con due palati, insomma non so nemmeno io come esprimermi. (ABATE, 2000, p. 333)

Il cuoco rimane legato alla tradizione arbëreshe senza tuttavia coltivare l'espressione purista della cucina: nell'affermare il "palato diviso" o i "due palati", egli non solo riflette la complessità dei rapporti tra il migrante e la società di accoglienza, i meccanismi di appartenenza e identificazione, ma anche la definizione di un'identità fluida. L'adattamento di certe preparazioni alimentari fuori dal loro contesto originario, a contatto con nuove abitudini e riferimenti ("scambio"), richiede la necessità di una costante rielaborazione o ridefinizione, rispetto alla tendenza opposta ("identità") 
della conservazione ad ogni costo della tradizione alla quale non si possono fare concessioni affinché evitare il pericolo della contaminazione.

Il cuoco d'Arbëria riunisce insieme per l'io narrante la possibilità della convivialità, della condivisione del pasto, il bisogno di comunione che si realizza attraverso il mangiare insieme (in questo senso anche i pasti al centro italiano assolvono a questa funzione), e una risposta al bisogno di riconoscersi per ritrovare il proprio luogo.

In Germania, il cuoco d'Arbëria si era ricreato una specie di luogo originario ideale:

In quei quaranta metri quadrati, tra casetta di legno e orto, in mezzo ai pomodori peperoncini cetrioli, si sentiva a casa sua. Per questo non provava nostalgia, per questo tutto il resto poteva stare comodamente dentro di lui, quando zappava la terra della "villa" come fosse la "sua" terra. (ABATE, 2000, p. 334)

Per Vito Teti, la ricreazione dei luoghi originari può essere considerata un "doppio": doppio del paese, doppio dell'orto del paese. La tradizione del paese d'origine (il "paese uno") viene rifondata nel paese d'arrivo (il "paese due"). Questo fatto non solo promuove il mantenimento dei legami con la terra d'origine, ma favorisce anche lo scambio attraverso la traduzione, l'ibridazione e le pratiche di sincretismo che ivi hanno luogo.

Lo scambio del cibo tra i "due paesi" attesta il suo valore fortemente identitario e si apre alla concezione di un'identità fluida, mobile e aperta che secondo Montanari si fanno più forti se inserite in percorsi di scambio e contaminazione (MONTANARI, 2002, p. XII).

La formazione di una cucina a seguito di processi immigratori segue percorsi diversi. I popoli che si spostano si fanno carico delle proprie abitudini e tradizioni alimentari. Per soddisfare la pratica di queste tradizioni portano nel bagaglio piante, spezie, ricettari, ma anche preferenze, divieti, associazioni ed esclusioni. Mescolando o aggiungendo alla propria cucina nuove possibilità e pratiche alimentari della terra di accoglienza, creano cucine con caratteristiche particolari. In questo senso la cucina riflette i processi di continua elaborazione del cambiamento, in una ridefinizione 
dell'identità, che sono alla base del processo migratorio.

La relazione tra il cibo e l'identità per il migrante può essere definita a grandi linee su due assi importanti. Da una parte si prospetta la salvaguardia dei principi identitari fondati sulla purezza, sul ritorno ad una unità originale e al mantenimento della tradizione e saperi, che può sfociare, come nel racconto Curry di pollo in un atteggiamento di separatezza ed esclusione; dall'altra, si delinea la formazione delle identità ibride, del meticciato culturale, dell'apertura al sintagma identitario plurimo, rappresentato dalle patate del cuoco d'Arbëria. Questi due sistemi identitari s'identificano con la dialettica delle identità di Stuart Hall, sintetizzate nel binomio tradizione/traduzione, in cui la prima si riferisce alla chiusura alla contaminazione e al desiderio di restaurare la purezza originale; la seconda punta invece all'attraversamento di frontiere e alla formazione di nuove identità in un processo di continua negoziazione. Il concetto di traduzione non cancella il legame con la terra d'origine, ma esclude l'illusione di un ritorno al passato; esso implica un processo di negoziazione con le nuove culture con le quali l'individuo dislocato viene a contatto, che non equivale, però, alla perdita dell'identità o alla sua assimilazione dalla parte di esse. La cultura tradotta è il risultato dell'intersecazione tra diverse storie e culture, ma mantiene le tracce della cultura originaria, delle tradizioni, del linguaggio e delle storie che ne caratterizzano la fisionomia. D'altro canto, il tentativo di ricostruire la purezza identitaria originaria, di restaurare la coesione e la tradizionepuò essere una risposta all'esperienza del razzismo e dell'esclusione, contrastati dalla ridentificazione con la cultura d'origine, dal revival del tradizionalismo culturale, dell'ortodossia religiosa e del separatismo politico (HALL, 1992, pp. 307-309).

Il cibo è un forte elemento d'identificazione del migrante con la terra d'origine; per mezzo della tavola, che rappresenta sia i cibi perduti sia la convivialità, si recuperano i tratti distintivi del mondo originario perduto, del luogo antropologico, si identificano i percorsi dell'appartenenza e si ridefinisce il sé. Questo processo, avverte Vito Teti, non può essere ridotto al semplice rimpianto per un determinato cibo piuttosto che per un altro; si tratta per l'individuo dislocato di un processo di recupero dei valori originali, individuali e culturali del mondo d'origine. 


\section{Referências bibliográficas}

ABATE, C. Il cuoco d'Arbëria. In: Nuovi Argomenti, 10: 328-338, 2000.

AUGÉ, M. Nonluoghi: introduzione a una antropologia della surmodernità. Trad. D. Rolland. Milano: Eléuthera, 2000.

BIASIN, G. P. Gelato e peperoncino. In: Forum Italicum, 29, 1: 103-113, 1995.

CHAMBERS, I. Paesaggi migratori: cultura e identità nell'epoca postcoloniale. Trad. A. Biavasco e V. Guani. Genova: Costa \& Nolan, 1996.

CHOHRA, N. Volevo diventare bianca. (org. A. Atti di Sarro). Roma: e/o, 1993.

COOK, I. e CRANG, P. The World on a plate: culinary culture, displacement and geographical knowledge. In: Journal of Material Culture, 1, 2: 131-153, 1996.

DOUGLAS, M. Deciphering a meal. In: Implicit meanings: selected essays in Anthropology. 2. ed. New York: Routledge, 1999, pp. 231-251.

HALL, S. The Question of Cultural Identity. In: HALL, S., HELD, D., MCGREW, T. (org.). Modernity and its Futures. Cambridge: Polity Press, 1992, pp. 274-325.

HORN, V. La conquista dell'Italia e la questione dell'altro: letteratura, migrazione e canone nell'Italia attuale. Tese. (Dottorato di ricerca in Studi Italianistici) - Università degli Studi di Pisa, Pisa, ciclo 2005-2007.

LAKHOUS, A. Scontro di civiltà per un ascensore a Piazza Vittorio. Roma: e/o, 2006.

MONTANARI, M. Il cibo come cultura: Roma-Bari: Laterza, 2004. . La cucina, luogo dell'identità e dello scambio. In: (org.). Il mondo in

cucina: storia, identità, scambi. Roma-Bari: Laterza, 2002, pp. VII-XII.

SCEGO, I. Salsicce. In: SCEGO, I. et al. Pecore nere . Roma-Bari: Laterza, 2006, pp.23-36.

TETI, V. Emigrazione, alimentazione, culture popolari. In: BEVILAQUA, P., DE CLEMENTI, A., FRANZINA, E. (org.). Storia dell'emigrazione italiana. Roma: Donzelli, 2001, pp. 575-597.

. Il colore del cibo: geografia, mito e realtà dell'alimentazione mediterranea. Roma: Meltemi, 1999.

WADIA, L. Curry di pollo. In: SCEGO, I. et al. Pecore nere. Roma-Bari: Laterza, 2006, pp. $39-52$. 